\title{
RSSI Measurements for RFID Tag Classification in Smart Storage Systems
}

\author{
Alice Buffi ${ }^{1}$, Andrea Michel ${ }^{2}$, Paolo Nepa ${ }^{2}$, Bernardo Tellini ${ }^{1}$ \\ ${ }^{1}$ Department of Energy, Systems, Territory and Constructions Engineering, University of Pisa, IT-56122 Pisa, Italy \\ ${ }^{2}$ Department of Information Engineering, University of Pisa, IT-56122 Pisa, Italy
}

\begin{abstract}
This paper presents a measurement method for tagged item classification in radio frequency identification (RFID)-based smart drawers. Specifically, the amplitude of the signal backscattered by the UHF-RFID passive tags attached to the items inside the drawer is used to identify at which of an assigned set of drawer subregions each detected tag belongs. The received signal strength indicator (RSSI) may be acquired by almost any UHF-RFID commercial reader chipset compatible with the Electronic Product Code (EPC) Class1 Gen2 protocol. The RSSI model in terms of the position of the tag inside the drawer is introduced, and its selective properties are discussed through both numerical data and measurements. The cases of a single reader antenna measuring during natural opening/closing operations as well as of multiple reader antennas measuring when the drawer is static are investigated. The measurement method performance is shown through an experimental analysis in a realistic scenario, where the RSSI values are collected by using commercial UHF RFID hardware components, and then used as the feature vector of the classification algorithm. As an example, two different classifiers are used to map each detected tag to one of the regions the drawer is subdivided into. The classification performance of the method based on a single antenna exploiting the drawer natural sliding movements is comparable to that achievable with multiple reader antennas, while representing a cost-effective and easy-to-implement practical solution.
\end{abstract}

Index Terms - Antenna near-field region, classification techniques, radio frequency identification (RFID) tag classification, received signal strength indicator (RSSI) measurements, RFID tag indoor localization, RFID-based smart drawers, RFID-based smart storage spaces, UHF-RFID measurements.

\section{INTRODUCTION}

I $\mathrm{N}$ the context of the Internet-of-Thing paradigm, the UHF radio frequency identification (RFID) technology is gaining increasing attention for item-level tagging in smart spaces [1] such as smart cabinets/shelves and smart fitting rooms [2]-[5].

In addition to automatic real-time inventory, the UHF-RFID passive technology can be used to implement low-cost radiolocalization techniques for item localization in indoor scenarios [6]-[10]. In this context, a measurement system locating a tagged object in shelves/drawers allows developing additional capabilities, as, for example, to avoid misplaced items in pharmaceutical or retail environments [11] (e.g. items left on the wrong shelf by the customers, products forbidden or lost in the fitting room).

Basic solutions suggest localizing tags based on their presence/absence within the reader antenna read range; thus, tag localization is possible just in a specific region within the area of interest and with a low-coarse grained resolution. Such a principle is that exploited in the HF system in [12], where a multi-antenna arrangement is applied to distinguish among tagged items within four separate regions inside a drawer. To apply the same approach at higher frequency (UHF band), the measurement system must be designed with ad hoc reader antennas, which can radiate a spatially confined electromagnetic field [13], [14], by exploiting the near-field coupling [15], [16] between the reader and tag antennas.

For a more accurate localization in UHF-RFID smart spaces, measurement systems exploit both amplitude [received signal strength indicator (RSSI)] [17] and phase [18] of the tag backscattering signal. Above measurements can be employed as in positioning techniques for wireless sensors networks [19], [20]. However, in scenarios where the tags are close to the reader antenna (namely, tags does not lie into the far-field region of the reader antenna) it is difficult to define a simple analytical pathloss model. Indeed, the RSSI behavior is significantly dominated by near-field coupling phenomena, which strongly depend on the specific antenna layouts and the obstacles located around the two antennas.

Rather than the tag spatial coordinates, in practical scenarios with a large amount of tags, it could be reasonable to determine just the spatial region the tag belongs to [21]. This represents a classification issue, analogously to that addressed for discriminating among moving and static RFID tags [22], for person activities recognition in ambient assisted living applications [23], for imaging recognition [24] or for smell recognition [25].

In such a framework, the basic idea of a new measurement method to determine the belonging region of tagged items within 
UHF-RFID smart drawers was presented in [26]. RSSI measurements were acquired during the drawer opening/closing actions and employed to extract some useful features for the tag classification procedure. In [26], preliminary results were presented showing the validity of the method for a two-region classification.

This paper presents a methodology to make more selective the approach suggested in [26] for tag classification in smart storage systems. The basic requirement for an effective classification of the tag belonging region is the creation of a set of different tag interrogation field distributions in the area of interest, so that the vector of the corresponding RSSI values can be used to setup the feature vector of a certain classification algorithm. A number of reader antenna configurations are considered, resembling the cases of multiple reader antennas with a static drawer and of a single reader antenna with a sliding drawer. The reliability of the adopted RSSI model is shown through experimentally results for a three-region classification, when the RSSI data are acquired by a commercial reader.

The paper is organized as follows. The RSSI model is introduced and discussed in Section 2. Then, the performance of the classification approach is described in Section III, when a set of reader antennas are used to acquire the RSSI in a static drawer scenario. The classification performance of the configuration exploiting a single reader antenna and the natural sliding movements of the drawer is presented in Section IV. Finally, concluding remarks are given in Section 5.

\section{RSSI MODEL}

In an RFID system based on passive (battery-less) tags [Fig. 1(a)], the reader sends an interrogation signal to the tag, which is also used to energize the tag itself. If the power absorbed by the tag is higher than the RFID chip sensitivity, the tag is excited and it is able to send back its unique identifier through a backscattering modulation principle [27]. Specifically, the backscattered signal is modulated by changing the impedance load of the tag antenna between two different states, which usually correspond to a conjugate matching impedance load $\left(\mathrm{Z}_{\mathrm{CM}}\right)$ and a short circuit $\left(\mathrm{Z}_{\mathrm{SC}}\right)$ [28]. At the reader side, the amplitude of the demodulated signal is made available by the RSSI.

Let us consider a 2-D drawer scenario where the tag is located, with a reader antenna positioned at a certain point along one of the drawer sides. The coordinate pair $(x, y)$ allows to indicate the tag position with respect to the reader antenna, and $d$ is the reader-to-tag distance. By referring to the equivalent electric circuit in Fig. 1(b), the received signal strength at the reader side can be expressed in terms of the difference between the reflection coefficients corresponding to the two above tag impedance load states, $\Gamma_{C M}$ and $\Gamma_{S C}$. Actually, any commercial reader compliant with the standardized Electronic Product Code (EPC) Class1 Gen2 protocol makes available a digital value that is proportional to the power level of the received signal, which is known as the RSSI. Then, by denoting with $Z_{i n}$ the input impedance seen at the connector of the reader antenna and assuming that $K$ is a constant independent on the tag location, the RSSI can be expressed as:

$$
R S S I=K\left|\Gamma_{C M}(x, y)-\Gamma_{S C}(x, y)\right|^{2}
$$

with

$$
\Gamma=\frac{b_{1}}{a_{1}}=\frac{Z_{\text {ing }}-R_{0}}{Z_{\text {ing }}+R_{0}}
$$

where $R_{0}$ is the characteristic impedance of the reader antenna feeding line, $b_{1}$ and $a_{1}$ are the complex amplitudes of the reflected and incident voltage waves, respectively, at the reader antenna port. The reflection coefficient $\Gamma$ depends on the tag antenna load and on the electromagnetic coupling between the tag and reader antennas. Since in the drawer scenario the tag cannot be assumed as being in the far-field region of the reader antenna, the Friis formula for evaluating the differential radar cross section [28] does not work. The latter assumes a path-loss law of $-40 \mathrm{~dB} / \mathrm{decade}$ that is not valid in the antenna near-field region.

Therefore, using a full-wave numerical model is mandatory to estimate the mutual coupling between tag and reader antennas. Specifically, any numerical tool able to solve the Maxwell's equations with the proper boundary conditions can be used to derive the impedance matrix (3) of the two-port system made by the two antennas (modelled with the actual size and components) and any object and obstacle that could be located inside the region among them:

$$
\left[\begin{array}{l}
V_{1} \\
V_{2}
\end{array}\right]=\underline{\underline{Z}}\left[\begin{array}{l}
I_{1} \\
I_{2}
\end{array}\right]=\left[\begin{array}{ll}
Z_{11} & Z_{12} \\
Z_{21} & Z_{22}
\end{array}\right]\left[\begin{array}{l}
I_{1} \\
I_{2}
\end{array}\right]
$$

with $Z_{12}=Z_{21}$ being the network reciprocal. The impedance matrix entries of the two-port network can be numerically evaluated for any position of the tag inside the drawer. It is worth underlying that by definition the impedance matrix is independent on the tag antenna load. Then, the input impedance at the reader side can be evaluated, depending on the tag impedance load state

$$
Z_{\text {in }}=\frac{V_{1}}{I_{1}}=Z_{11}-\frac{Z_{12}^{2}}{Z_{22}+Z_{\text {chip }}}
$$

where $\mathrm{Z}_{\text {chip }}=\mathrm{Z}_{\text {tag }}$ * (the chip input impedance is equal to the conjugate of the tag antenna input impedance to maximize the power absorbed by the chip or $Z_{\text {chip }}=0$ (the short circuit loading allows to maximize the amplitude of the tag backscattered signal). 

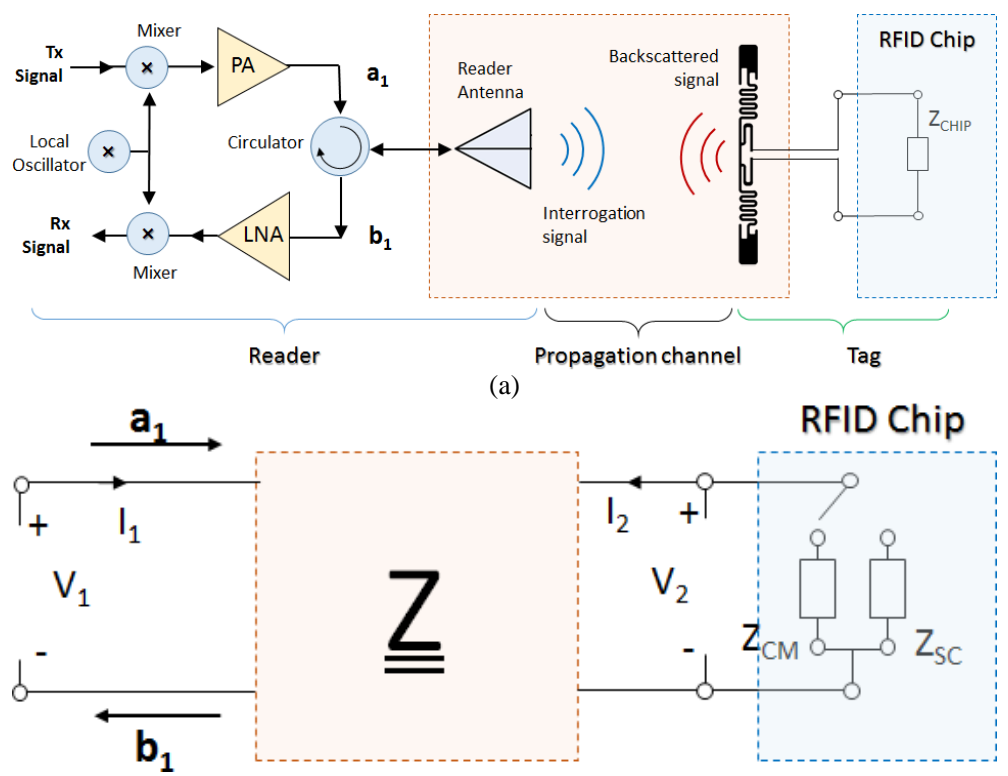

(b)

Fig. 1. (a) Schematic representation of a tag equipped with an antenna, an RFID chip, and a reader, the latter consisting of an antenna, a circulator, a power amplifier for the transmitted signal (forward link) and a low noise amplifier for the received signal (backward link). (b) Equivalent impedance matrix representing the reader antenna, the tag antenna, and the propagation channel between them. To generate a modulated backscattering, the tag antenna is connected to either a conjugate matching impedance load $\left(\mathrm{Z}_{\mathrm{CM}}\right)$ or a short circuit $\left(\mathrm{Z}_{\mathrm{SC}}\right)$.

Let us consider the interrogation field radiated by a reader antenna when it is located at different positions along the longer side of a drawer (Fig. 2). Depending on its position, the antenna excites a different electric field distribution within the drawer. As an example, a numerical analysis of the field radiated by the reader antenna was carried out by considering an $80 \mathrm{~cm} \times 40 \mathrm{~cm}$ drawer and a square patch antenna. The latter is a quite typical antenna layout used in commercial UHF RFID systems. The commercial software CST was been used to design the patch antenna as well as the get the field radiated by the antenna inside the drawer region. The patch antenna is printed on a 1.53-mm-height FR-4 substrate (relative dielectric permittivity $\varepsilon_{r}=4.4$ ) and its resonating side is $8.3 \mathrm{~cm}$ long, which corresponds to around half wavelength at the operating frequency of $f_{0}=865.7 \mathrm{MHz}$.

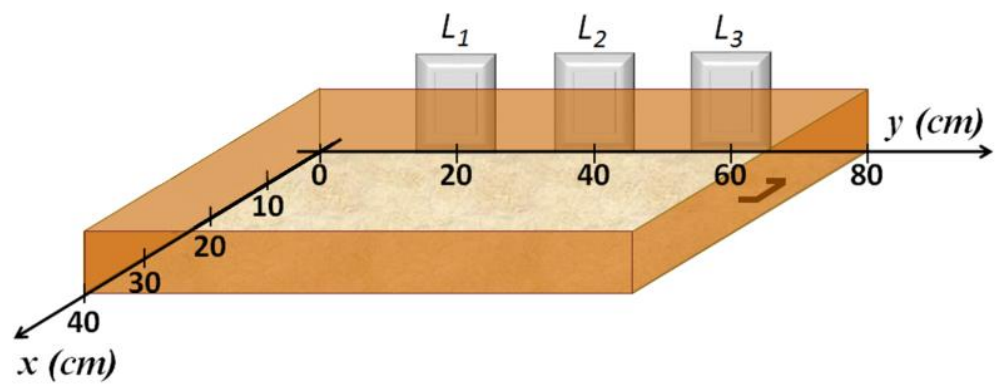

Fig. $2.80 \mathrm{~cm} \times 40 \mathrm{~cm}$ drawer with the reader antenna placed at $L_{1}(x=0 \mathrm{~cm}, y=20 \mathrm{~cm})$ or $L_{2}(x=0 \mathrm{~cm}, y=40 \mathrm{~cm})$ or $L_{3}(x=0 \mathrm{~cm}, y=60 \mathrm{~cm})$. Note that the origin of the coordinate system is at the left up corner of the drawer.

The relevant electric field distribution is represented in Fig. 3(a) and (b), when the reader antenna is located at positions $L_{I}$ $(x=0 \mathrm{~cm}, y=20 \mathrm{~cm})$ and $L_{2}(x=0 \mathrm{~cm}, y=40 \mathrm{~cm})$. A remarkable variation of the field amplitude along the $y$-direction is apparent, while the variation along the $x$-direction is slower. Since the amplitude of the tag backscattered signal is proportional to the amplitude of the incident field radiated by the reader antenna, this suggests that the acquired RSSI values relevant to a set of different reader antenna positions (each one creating a different field distribution within the area of interest) could be used as the feature vector of a classifier for determining the belonging region of a tag within the drawer. 


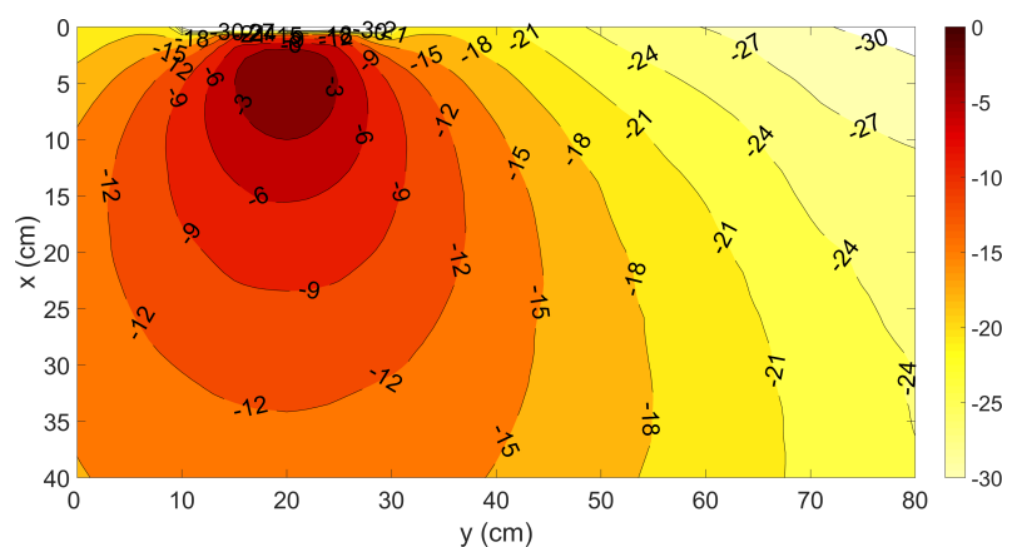

(a)

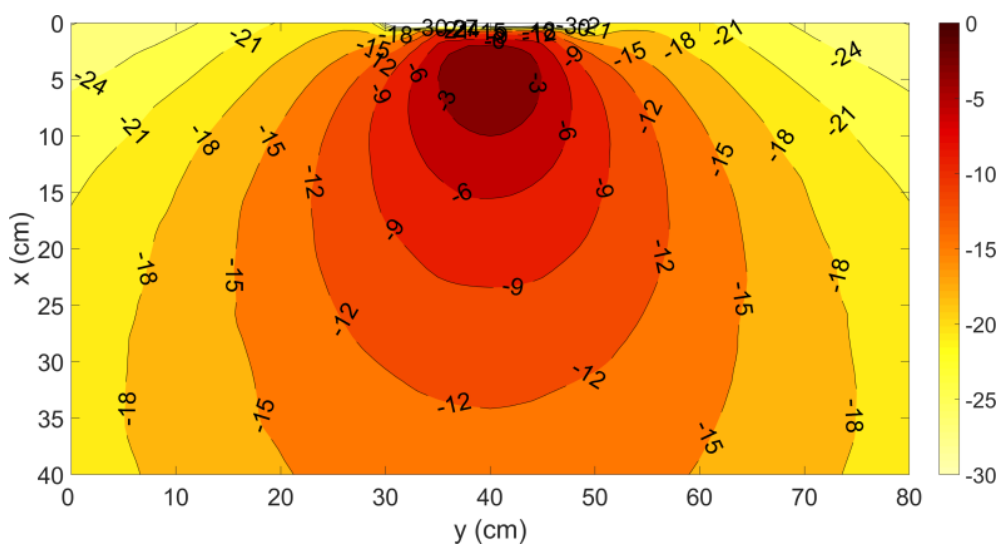

(b)

Fig. 3. Normalized electric field $(\mathrm{dB})$ radiated by a square patch reader antenna placed at different positions of the drawer scenario in Fig. 2 . (a) $L_{l}(x=0 \mathrm{~cm}$, $y=20 \mathrm{~cm})$. (b) $L_{2}(x=0 \mathrm{~cm}, y=40 \mathrm{~cm})$.

For a better understanding of the RSSI behavior for different positions of the tag with respect to the reader antenna, the differential reflection coefficient appearing in (1) was evaluated by considering the above reader patch antenna and a commercial tag: the dipole-like UH100 from LAB-ID [Fig. 1(a)]. The elements of the impedance matrix of the equivalent model in Fig. 1b were calculated by the commercial software CST. Such a full-wave simulation tool can account for any orientation and relative position of the tag antenna with respect to the reader antenna, as well as for any obstacle or item located within the space around the two antennas. It is worth noting that the adopted RSSI model is valid for any distance between the two antennas, even when such a distance is as small as a few millimeters [29]. The numerical results for the differential reflection coefficient in (1) (namely, the RSSI normalized to the constant $K$ ) were evaluated versus the reader-to-tag distance, $d$, and for a set of different directions from the antenna, and they are shown in Fig. 4 (see the geometry of the problem sketched into the inset of Fig. 4). It is worth noting that the normalized RSSI approaches the trend $1 / d^{2}$ (corresponding to a linear behavior when a $\log$ scale is used along both axes, with a $-40 \mathrm{~dB} / \mathrm{decade}$ slope) only when the reader-to-tag distance is larger than the free-space wavelength (around $33 \mathrm{~cm}$, at the UHF-RFID ETSI band of 865-868 MHz). Indeed, when the reader-to-tag distance is less than around one wavelength, the tag is located in the near-field region of the reader antenna, where the radiated electric field structure is quite complex and includes terms proportional to $1 / d, 1 / d^{2}$ and $1 / d^{3}$ [30]. On the contrary, at larger distances from the reader antenna, namely, into the far-field region of the reader antenna, the radiated electric field can be approximated with a local plane wave whose amplitude decreases as $1 / d$. In this case, the RSSI amplitude in (1) (which is a power level) will be proportional to $1 / d^{4}$, as both the forward link (reader to tag) and backward link (tag to reader) must be considered [31]. Moreover, if the tag is in the far-field region, the RSSI can be easily expressed in terms of the gain of the reader and tag antennas, through a conventional radar equation including the differential radar cross section [28].

Several indoor localization techniques were developed where an RSSI model (or path-loss model) proportional to $1 / d^{4}$ was assumed [32]-[34]. In more sophisticated models also accounting for the effect of multipath phenomena, a more general $1 / d^{n}$ model has been assumed where the path-loss exponent $n$ is heuristically estimated on the basis of extended measurement campaigns or numerical simulations. Unfortunately, as demonstrated by the numerical results in Fig. 4, above simple RSSI analytical models are useless in RFID-based smart storage spaces, as the distance $d$ cannot be considered larger than the freespace wavelength. It follows that tag localization cannot be performed through a deterministic approach, and statistical classification techniques can be a valuable alternative. 


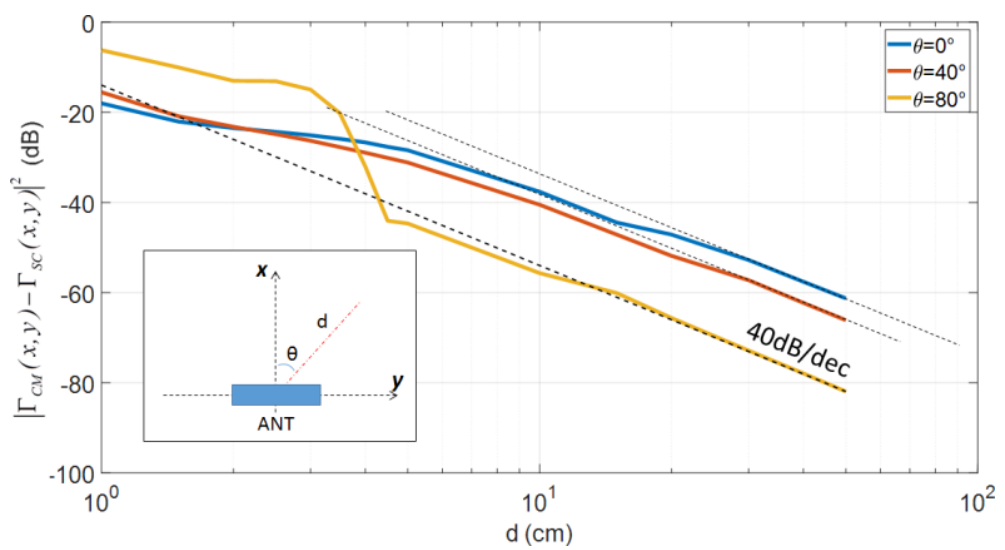

Fig. 4. Amplitude of the differential reflection coefficient in (1) as a function of the antenna-tag distance $(d)$ for different directions of the tag with respect to the reader antenna (angle $\theta$ defined in the inset).

The effectiveness of a classification method to determine the tag belonging region inside the drawer is related to the actual capability of getting different RSSI vectors for different tag positions. As an example, the amplitude of the differential reflection coefficient is shown in Fig. 5, for different positions of the tag with respect to the reader antenna (the reader antenna is at the origin of the coordinate system). A set of curves is obtained when the tag lies on different lines at distance $x$ from the reader antenna $(x=1,3,5,10,30,50 \mathrm{~cm})$, while the $y$-coordinate varies between $-40 \mathrm{~cm}$ and $+40 \mathrm{~cm}$. Even if the tag is located within the reader antenna near-field region, quite large variations of the normalized RSSI are evident as long as the reader-to-tag distance is less than $40-50 \mathrm{~cm}$, with a peak amplitude when the tag is at the minimum distance from the reader. For drawers larger than $40-50 \mathrm{~cm}$, an additional reader antenna can be added on the opposite side.

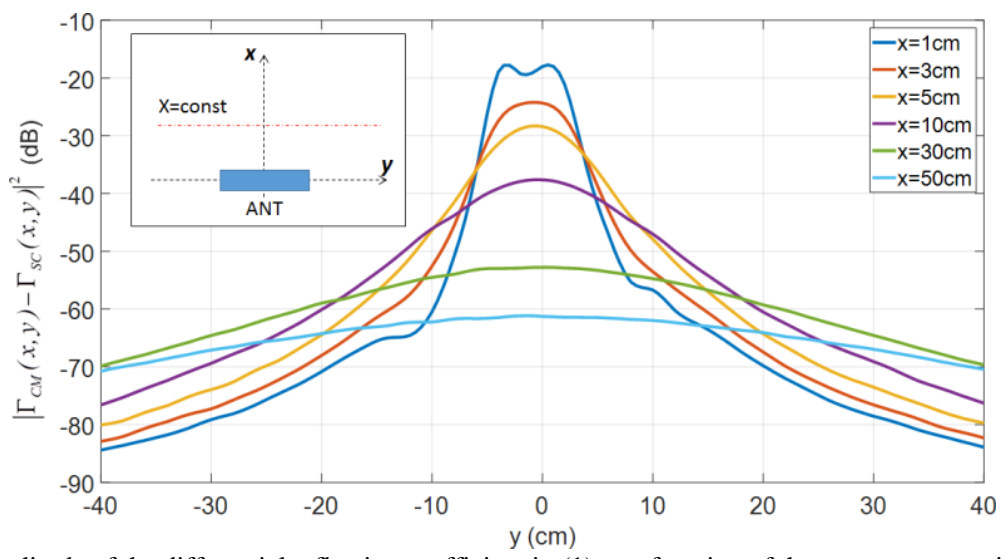

Fig. 5. Amplitude of the differential reflection coefficient in (1) as a function of the antenna-tag position $(x, y)$.

When referred to a $80 \mathrm{~cm} \times 40 \mathrm{~cm}$ drawer like that in Fig. 2, above results suggest that the belonging region of a tag inside the drawer can be estimated by using a set of RSSI values received by a number of reader antennas aligned along the longer drawer side. In a real implementation, a single reader can be used to sequentially feed all the antennas, as most of commercial RFID readers have a multiport output.

As an alternative implementation, a single antenna sliding along the drawer side can be used to profitably exploit the differential reflection coefficient variations in Fig. 5, namely the RSSI variations. A different RSSI profile will be acquired during the interrogation sequence, depending on the actual tag position inside the drawer. In a real implementation of the above technique, the relative movement between the single reader antenna and the tag can be obtained during the natural opening/closing operations of the drawer.

The classification performance that can be achieved by using a set of static reader antennas or a single reader antenna sliding with respect to the drawer are analyzed in Sections 3 and 4, respectively. Although the quite remarkable differential reflection coefficient variations shown in Fig. 5 would suggest an expected $100 \%$ overall accuracy by almost any basic classification algorithm, it is apparent that in real scenarios the performance can degrade due to several factors. Among them, it is worth mentioning the non-linearity of the tag chip, the interference from nearby tags, the effect on the backscattered signal of the material/shape of the tagged item, the multipath phenomena and the tag orientation with respect to the direction of the incident electric (polarization mismatching). In the RSSI model in (1), above effects can be modeled through an additive random variable. Some of the above mentioned random effects can be mitigated by averaging the acquired RSSI values, or by resorting to more 
sophisticated filters [35], [36].

\section{Classification with Multiple Static Antennas}

\subsection{Measurement Setup}

The RFID measurement setup was realized with the commercial reader R4300P ION by CAEN RFID. The reader is able to detect up to $200 \mathrm{tag} / \mathrm{s}$ and the conducted output power was set at $27 \mathrm{dBm}$. Experiments were performed in the ETSI RFID band (865-868 MHz). The measurement campaign was carried out in a real indoor scenario, thus the effects of environmental clutter and multipath propagation were present. However, it is expected that multipath does not affect significantly the method performance, since reader antenna and tags are very close each other.

Three linearly polarized reader antennas (model WANTENNAX007 by CAEN RFID) were considered. They were placed beside a wooden drawer of $80 \mathrm{~cm} \times 40 \mathrm{~cm}$, in positions $L_{1}, L_{2}$ and $L_{3}$ as shown in Fig. 6, and they are sequentially fed by the reader. The drawer was filled with forty-two drug boxes of different sizes (Fig. 7), each one tagged with a tag UPM Raflatac Rafsec G2.

The tags stand vertically, in a polarization matching condition with respect to the reader antennas, and they are uniformly distributed within the drawer.

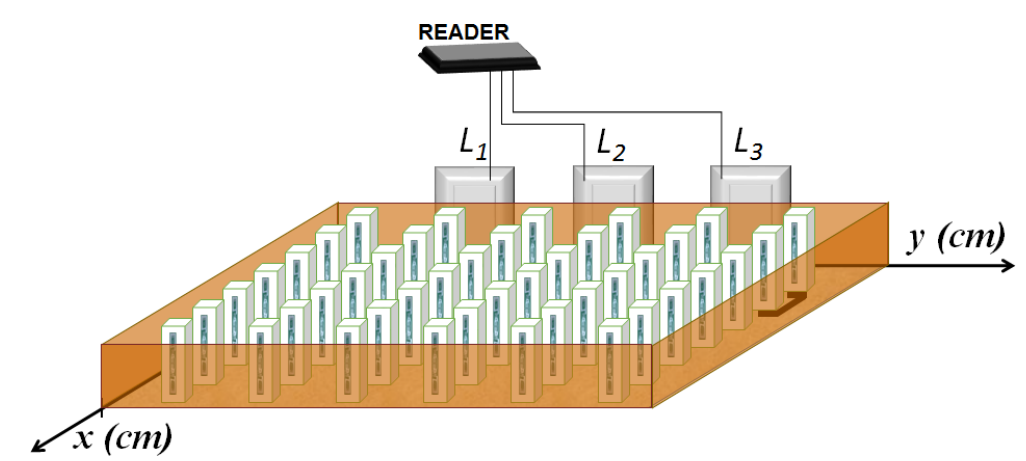

Fig. $6.80 \mathrm{~cm} \times 40 \mathrm{~cm}$ wooden drawer filled with forty-two tagged drug boxes arranged in a $7 \times 6$ grid. The three vertically polarized antennas located along the longer drawer side are sequentially fed by the reader.
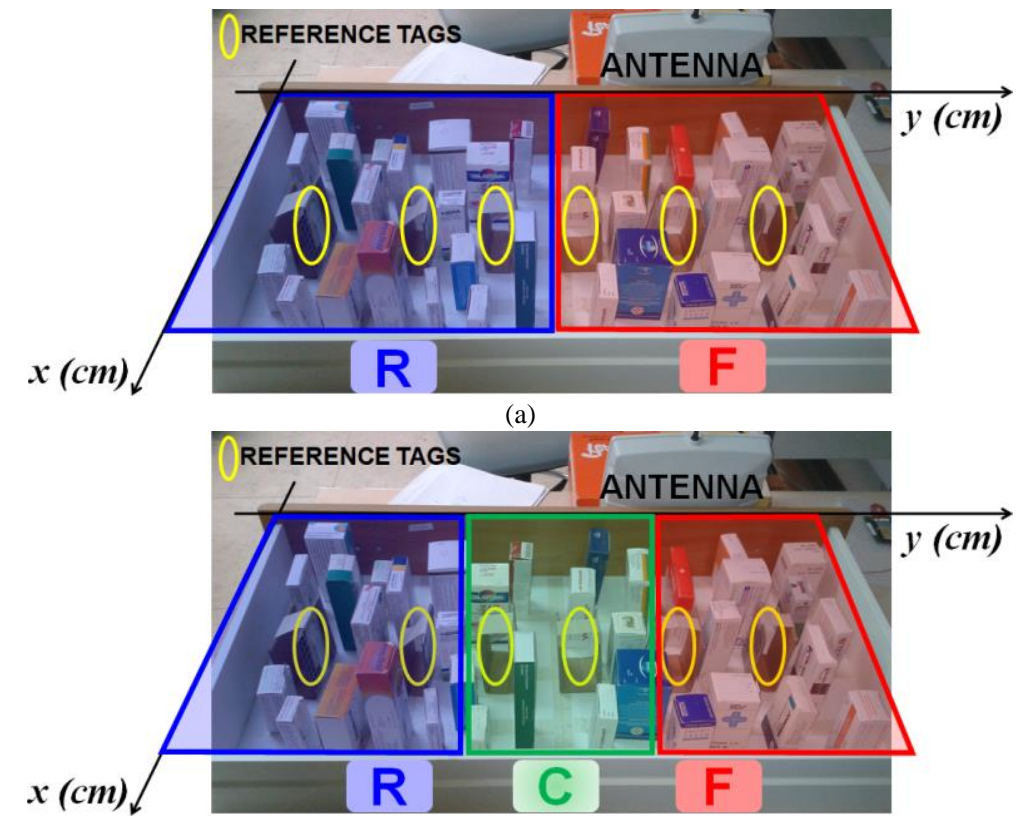

(b)

Fig. 7. Drawer scenario with forty-two tagged drug boxes subdivided into (a) $M=2$ and (b) $M=3$ drawer regions. The six reference tags can be noticed in the middle of the drawer.

For each useful tag reading (successful tag detection), the reader output logfile contains the EPC, which is the unique code identifying the tag, the index of the antenna that detected the tag, the reading time instant (timestamp) and the RSSI value expressed in $\mathrm{dBm}$. 


\subsection{RSSI Measurements}

The RSSI measured distribution within the drawer was investigated for a tag arrangement in a $7 \times 6$ grid as shown in Fig. 7 .

To filter out fluctuations due to thermal noise and other uncontrollable environment effects (such as varying multipath phenomena related to persons moving inside the room), an RSSI value averaged on 20 measured samples was considered for each tag, and they are shown in Fig. 8.

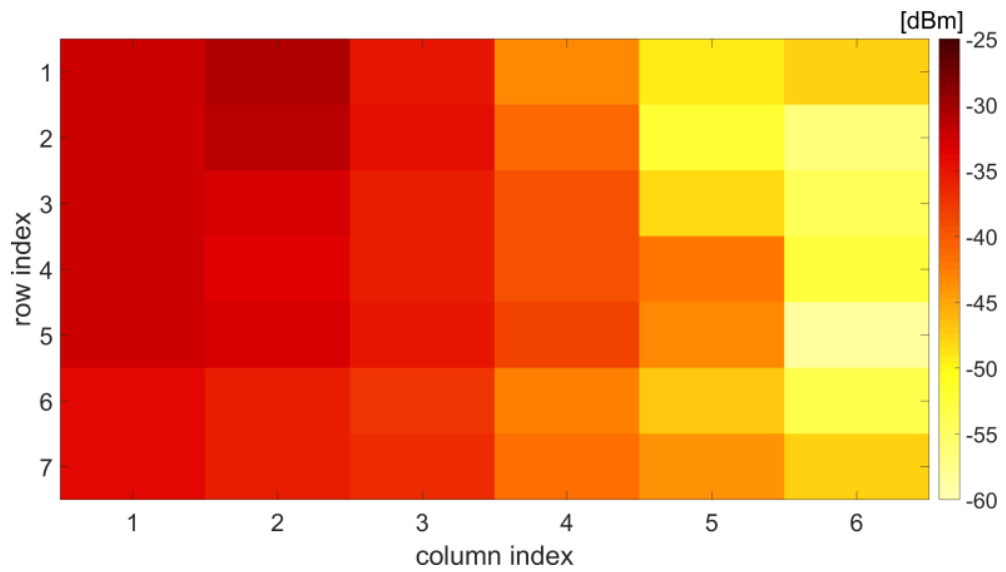

(a)

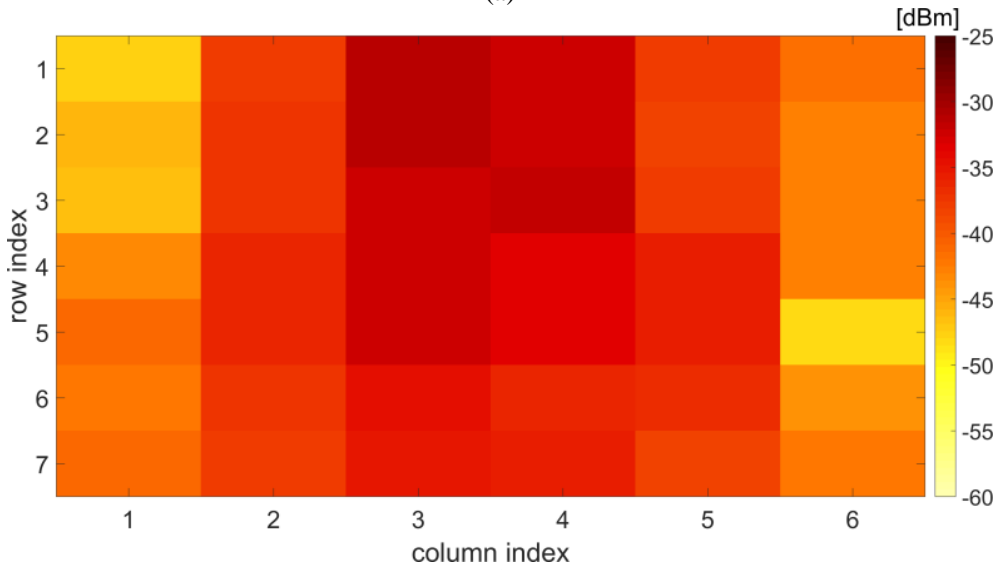

(b)

Fig. 8 RSSI $(\mathrm{dBm})$ distribution for a grid of $7 \times 6$ tags, as measured by a reader antenna located along the longer drawer side at $\left(\right.$ a) $L_{l}(x=0 \mathrm{~cm}, y=20 \mathrm{~cm})$ and (b) $L_{2}(x=0 \mathrm{~cm}, y=40 \mathrm{~cm})$.

The RSSI values measured by the antenna $L_{l}$ are relatively high for tags close to the antenna in the left area, then they gradually decrease for tags farther away from the antenna [Fig. 8(a)]. The RSSI values measured by antenna $L_{2}$ are high for tags in the central area and they decrease along the lateral directions [Fig. 8(b)]. Such measured RSSI distributions shows a behavior that matches with that can be argued from the simulated values in Fig. 5, while also accounting for the effect of the mutual coupling among nearby tags, the package material of the tagged item and the multipath phenomena of the real scenario.

\subsection{Scatterplot analysis}

A scatterplot analysis was carried out from the measured RSSI values, to explore the possibility for distinguish among tags belonging to $M=2$ regions (rear region $R$ and front region $F$ ) with 21 tags in each one [Fig. 7(a)]. By considering the RSSI data acquired by one antenna only, either the antenna in $L_{1}\left(\mathrm{RSSI}_{1}\right)$ or that in $L_{2}\left(\mathrm{RSSI}_{2}\right)$, a partial overlapping of the markers can be observed [(Fig. 9(a) and (b)]. By considering the whole RSSI data acquired by both antennas, $L_{1}$ and $L_{2}\left(\operatorname{RSSI}_{1}\right.$ and $\left.\mathrm{RSSI}_{2}\right)$, or $L_{1}$ and $L_{3}\left(\mathrm{RSSI}_{1}\right.$ and $\mathrm{RSSI}_{3}$ ), the point clouds start to separate [Fig. 9(c) and (d)]. A better discrimination can be obtained by employing RSSI values acquired by three antennas $\left(\mathrm{RSSI}_{1}, \mathrm{RSSI}_{2}\right.$, and $\left.\mathrm{RSSI}_{3}\right)$ as shown from the well distinct point clouds appearing in Fig. 9(e). 

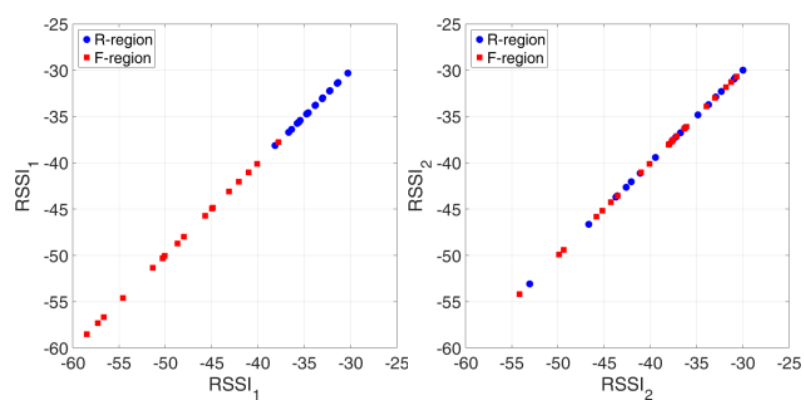

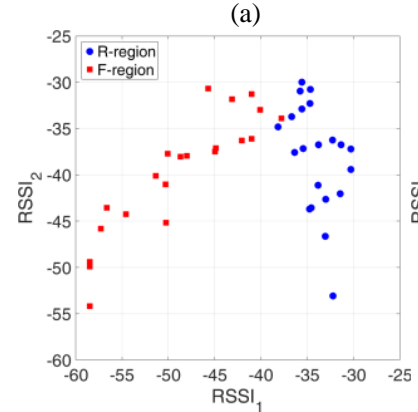

(c)

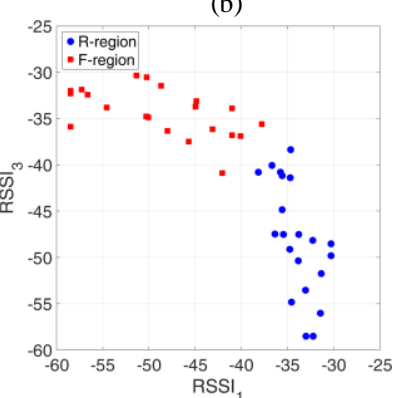

(d)

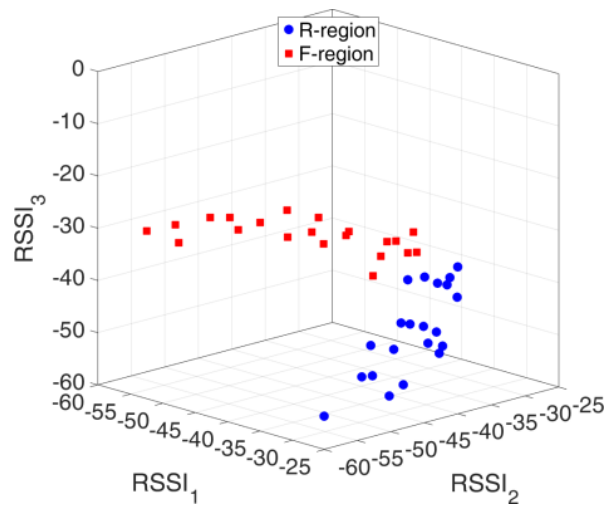

(e)

Fig. 9 Scatter plot of RSSI values measured at (a) antenna \#1, (b) antenna \#2, (c) antennas \#1 and \#2, (d) antennas \#1 and \#3, and (e) antennas \#1, \#2 and \#3, by using sequentially fed reader antennas beside the drawer. Forty-two tags are within the drawer, uniformly distributed in $M=2$ regions.

Similar considerations can be done by employing a drawer subdivision into $M=3$ regions (rear region $R$, central region $C$, and front region $F$ ), with 14 tags in each one [Fig. 7(b)]. Even in this case, it is possible to clearly distinguish three point clouds (Fig. 10) when employing all RSSI data acquired at three antennas.

Such analysis confirms the possibility of employing the RSSI measurements from multiple static antennas as features (input parameters) of a classification algorithm, to determine the region the tag belongs to.

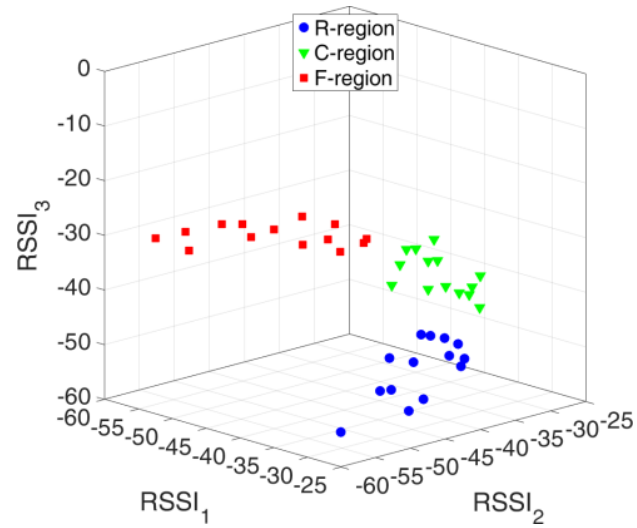

Fig. 10 Scatter plot of RSSI values measured at antennas \#1, \#2 and \#3, by using sequentially fed reader antennas beside the drawer. Forty-two tags are within the drawer, uniformly distributed in $M=3$ regions. 


\subsection{Classification method}

The possibility to distinguish among tags in a number of different drawer regions $M$, is here investigated for $N$ static antennas besides the drawer, by using $N$ measured RSSI values, namely $N$ classification features. In particular, $N=3$ RSSI values $\left(\mathrm{RSSI}_{1}\right.$, $\mathrm{RSSI}_{2}$, and $\mathrm{RSSI}_{3}$ ) were employed to classify tags in either $M=2$ drawer regions [ $R$ and $F$ regions in Fig. 7(a)] and $M=3$ drawer regions $[R, C$ and $F$ regions in Fig. 7(b)].

In order to show the effectiveness of the proposed classification technique, two well-known classification algorithms were applied: the linear discriminant analysis (LDA) [37] and the Naïve-Bayes algorithm [38].

The LDA method is a supervised classification algorithm [37], where classes are known a priori (the latter are the regions the drawer is subdivided into). For the training procedure, up to six reference tags were considered within the drawer, three for each region when a drawer division in $M=2$ drawer regions is considered [Fig. 7(a)], or two [Fig. 7(b)] when $M=3$ drawer regions are considered. Thus, the classifier employs RSSI samples measured from the reference tags to learn about class characteristics. It is noteworthy that this training process is done simultaneously with the classification process since both reference and target tags are detected during the same inventory phase. Therefore, a preliminary calibration procedure is not required.

System performance was evaluated in terms of the normalized confusion matrix. Each matrix element corresponds to the number of tags actually belonging to region $i$ and classified as belonging to the region $j$, normalized with respect to the total number of tags belonging to the region $i$. The diagonal terms of the confusion matrix represent the producer's accuracy [39], PA:

$$
\mathrm{PA}_{i}=\frac{\text { number of tags correctly assigned to region } i}{\text { number of tags belonging to region } i}
$$

Such parameter gives information about the classification accuracy for each class (i.e. drawer region). To get the overall accuracy of all classes OA, an average operation of the above producer's accuracies can be applied; since tags are equally divided into two/three regions (equal populations for each class), and the hypothesis of equal prior probability classes is assumed:

$$
\mathrm{OA}=\frac{\text { total number of correctly classified tags }}{\text { total number of tags }}
$$

Table 1 shows the normalized confusion matrix related to eigth repeated tests obtained by randomly changing the tag position inside the drawer; the region indexes are $\{F, R\}$ for the two-region drawer, and $\{F, C, R\}$ for the three-region drawer. When considering the case of $M=2$ drawer regions (three reference tags per region), an average overall accuracy $\overline{\mathrm{OA}}=0.88$ is reached (Table I) with a producer's accuracy $\mathrm{PA}_{R}=0.91$ and $\mathrm{PA}_{F}=0.85$, for the rear and front regions, respectively. On the other hand, when considering $M=3$ regions (with two reference tags per region), the average overall accuracy is $\overline{\mathrm{OA}}=0.81$ (Table II) and the worst producer's accuracy is that one of the rear region $\left(\mathrm{PA}_{R}=0.69\right)$.

TABLE I

Normalized Confusion Matrix Related to the Static Drawer MEASUREMENT SETUP IN Fig. 7. (a) (ThIRTY-SiX TARGET TAGS AND SiX REFERENCE

\begin{tabular}{|c|c|c|c|}
\hline & & \multicolumn{2}{|c|}{ Predicted class } \\
\hline & & $F$ & $R$ \\
\hline Actual & $F$ & 0.85 & 0.15 \\
\hline class & $\boldsymbol{R}$ & $\begin{array}{l}0.09 \\
\end{array}$ & 0.91 \\
\hline
\end{tabular}
TAGS) AS RESULT OF THE LDA ALGORITHM FOR EIGHT REPEATED TESTS:

TABLE II

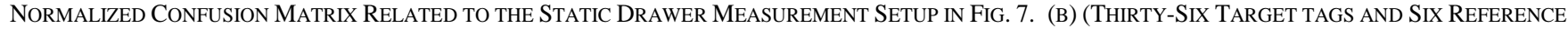
TAGS) AS RESUlt OF THE LDA ALGORITHM FOR EIGHT REPEATED TESTS:

\begin{tabular}{lcccc}
\hline \multicolumn{5}{c}{$N=3$ FEATURES AND $M=3$ DRAWER REGIONS } \\
\hline & \multicolumn{4}{c}{ Predicted class } \\
\hline & $\boldsymbol{F}$ & 0.84 & 0.15 & 0.01 \\
\cline { 2 - 5 } \multirow{2}{*}{$\begin{array}{c}\text { Actual } \\
\text { class }\end{array}$} & $\boldsymbol{C}$ & 0.08 & 0.90 & 0.02 \\
\cline { 2 - 5 } & $\boldsymbol{R}$ & 0.21 & 0.10 & 0.69 \\
\hline \hline
\end{tabular}

Besides, a Naïve-Bayes classifier algorithm was considered, which is a supervised classifier based on applying Bayes' theorem [38]. This algorithm was tested for the static scenario as for the LDA algorithm, with 36 target tags uniformly distributed inside the drawer and six reference tags, three for each region (the classifier was here applied only to the two regions case $M=2$ ). The same RSSI data sets as for the LDA algorithm performance were used. The classification performance is 
shown in Table III. For the case with $M=2$ drawer regions, an average overall accuracy $\overline{\mathrm{OA}}=0.875$ can be reached, with a producer's accuracy equal to $\mathrm{PA}_{R}=0.84$ and $\mathrm{PA}_{F}=0.91$, for the rear and front regions, respectively (Table III).

TABLE III

Normalized Confusion Matrix Related to the Static Drawer Measurement Setup in Fig. 7(a) (ThiRTY-Six TaRget TagS and Six RefERenCe TAgs) AS RESUlt OF NAÏVE-BAYES ALGORITHM FOR Eight REPEATED TESTS:

$N=3$ FEATURES AND $M=2$ DRAWER REGIONS.

\begin{tabular}{cccc}
\hline \hline & & \multicolumn{2}{c}{ Predicted class } \\
\hline & & $\boldsymbol{F}$ & $\boldsymbol{R}$ \\
\hline Actual & $\boldsymbol{F}$ & 0,91 & 0,09 \\
\cline { 2 - 4 } class & $\boldsymbol{R}$ & 0,16 & 0,84 \\
\hline \hline
\end{tabular}

Although just a basic implementation was considered for both classification algorithms, the overall accuracy achieved with the two procedures confirms the selectivity of the measurement method by using sequentially fed reader antennas besides the drawer.

\section{Classification With a Single Antenna AND the Sliding DraWer}

The measurement setup with the sliding drawer was realized with the same hardware as for the static configuration described in Sect. III, but only one reader antenna was used (Fig. 11). To get enough RSSI measurements, the drawer sliding natural action was exploited, as the distance between the reader antenna and the tags varies during the opening/closing operation. The use of a sliding drawer was already proposed in an HF-RFID system [40], but it was applied just to improve the inventory performance, namely to detect all the tags inside the drawer, and not for tagged item classification.

The antenna was placed at the front part of the drawer, at around $y=70 \mathrm{~cm}$ [Fig. 11(a)]. During the opening action, all tags pass in front of the reader antenna; the configuration with the drawer completely open is illustrated in Fig. 11b. The antenna relative displacement is of around $60 \mathrm{~cm}$ along the $y$-axis. The drawer motion was made with different sliding times (or sliding speeds).

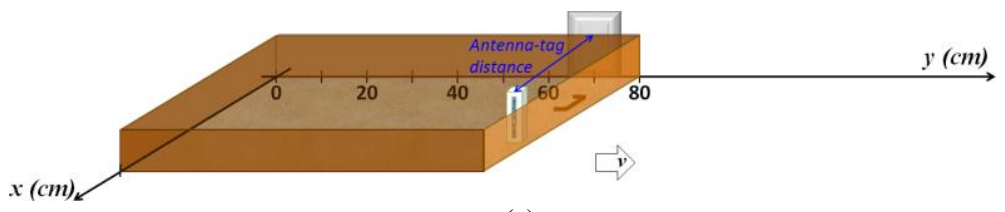

(a)

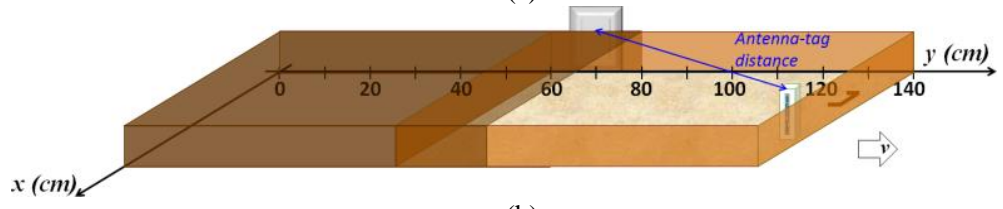

(b)

Fig. 11 Measurement setup with one reader antenna placed beside the sliding drawer. Antenna position with respect to (a) the closed drawer and (b) the drawer completely open.

During the drawer sliding action, the measured RSSI data vary according to the distance variation between the reader antenna and the tags (both target tags and reference tags). As an example, the RSSI samples measured for tags belonging to region $F$, $C$ and $R$ are represented in Fig. 12, with respect to the acquisition time, for an opening time of $T=13 \mathrm{~s}$.

The number of successful readings is not the same for all the tags within the drawer, as shown in Table IV for all thirty-six tags in the scenario. Thus, for each detected tag, an equal number $N$ of valuable parameters should be extracted from the measured RSSI samples (Fig. 12) to get $N$ useful features for the classification algorithm. This was accomplished by splitting the sliding interval $T$ into $N$ subintervals $\Delta t=T / N$ and by averaging the measured RSSI samples in each subinterval. The values of the RSSI samples averaged by considering $N=3$ subintervals are also shown in Fig. 12 (solid line). The aim of the averaging operation is twofold, as it also allows for filtering out unpredictable RSSI fluctuations. It is apparent the different behavior for tags belonging to different regions. For the tag that lies within the $F$-region, the $N=3$ features assume decreasing values since the tag moves away from the antenna. For the tag in the $R$-region, the feature values are increasing since the tag approaches the antenna. Instead, for the tag in the $C$-region, the highest feature value is achieved when the tag passes in front of the reader antenna, roughly in the middle of its path.

It is noteworthy that collecting $N$ averaged RSSI samples for each tag during drawer sliding looks like getting RSSI measurements with $N$ antennas distributed along the side of a static drawer (see Sect. 3). Then it is expected that the algorithm performance depends on the value chosen for the temporal subinterval, $\Delta t$, namely the number of corresponding algorithm 
features, $N$.

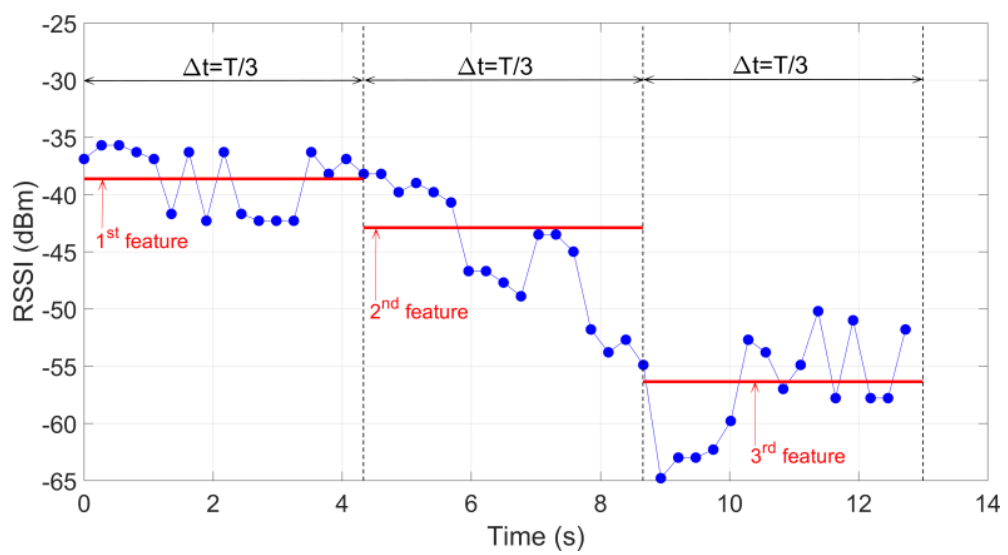

(a)

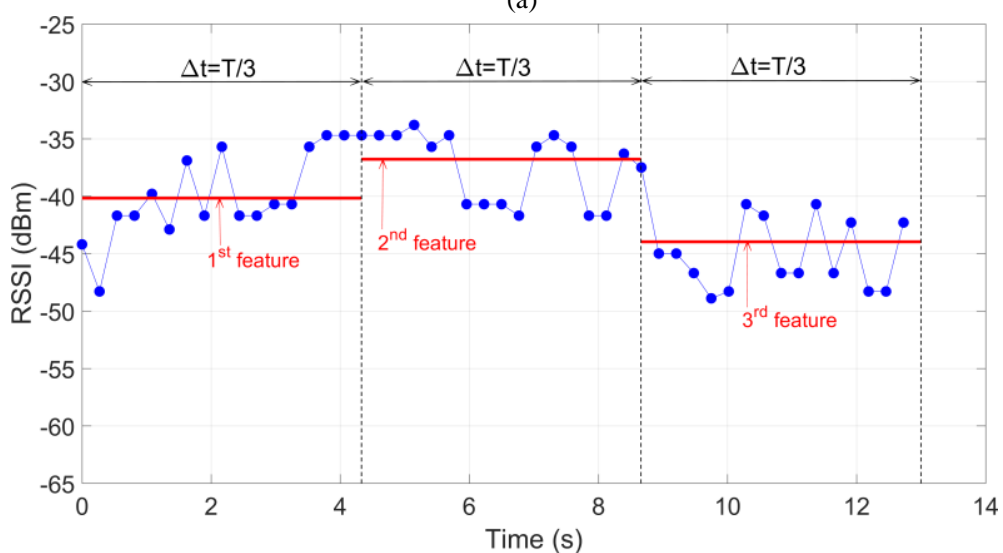

(b)

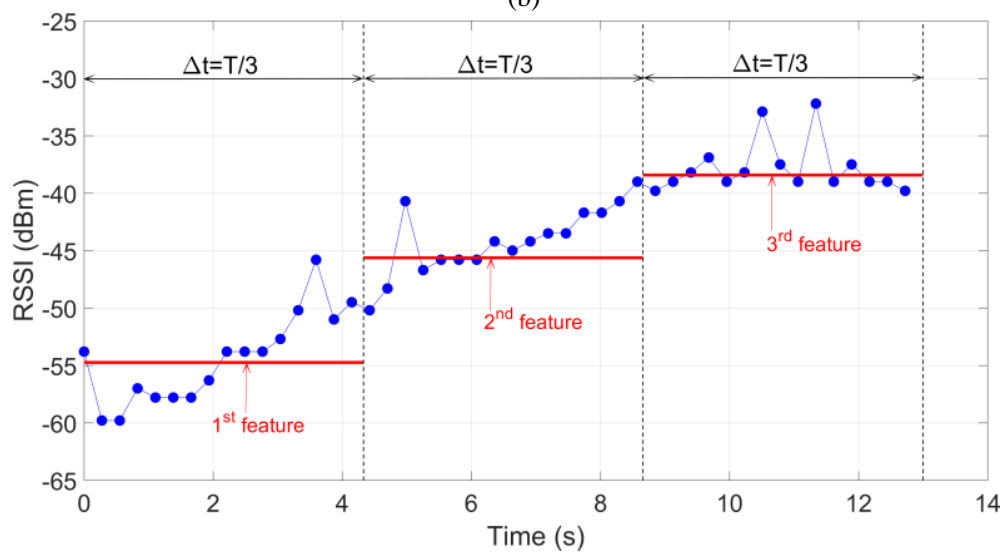

(c)

Fig. 12 RSSI values (dBm) measured during an opening action for a tag located in (a) front region, (b) central region, and (c) rear region. In each subinterval, the constant value is the average value of the acquired RSSI samples.

Depending on the value chosen for the time subinterval $\Delta t$ and the sliding speed, some tags could be not identified within all subintervals, giving rise to a different number of available features for each tag. In Table V, the number of tags that were detected in all subintervals are shown for a measurement scenario with forty-two tags, for different values of $\Delta t$ and a drawer sliding time $T=5 \mathrm{~s}$. By choosing $\Delta t=T / 3$, it is possible to detect all tags within the drawer (both reference and target tags) in all subintervals, and at least $N=3$ features are available for each tag. By decreasing $\Delta t$, the number of tags that are detected in all subintervals decreases. This suggests that depending on the sliding time $T$ (tied to the drawer length and opening/closing speed) the value of the time subinterval $\Delta t$ has to be chosen in such a way to guarantee an equal number $N$ of RSSI samples available for all tags within the drawer. Among such values of $\Delta t$, the interval that allows for the largest number of available RSSI samples for all tags is selected. A new scanning procedure is required, until at least $N=2$ algorithm features are available for each tag. 
TABLE IV

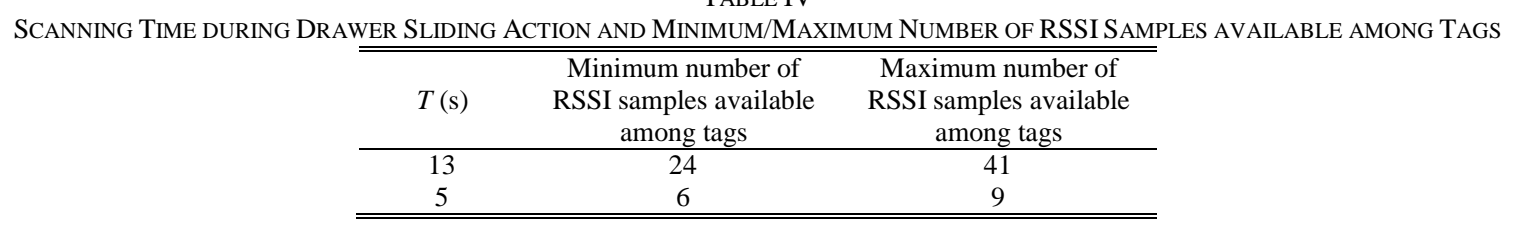

TABLE V

Number of Detected TAgs in All Subintervals, For VARIOUS Time Subintervals $\Delta t=T / N$, FOR the CASE $T=5 \mathrm{~s}$.

\begin{tabular}{ccc}
\hline \hline$N$ & $\begin{array}{c}\text { Number of detected } \\
\text { reference tags }\end{array}$ & $\begin{array}{c}\text { Number of detected } \\
\text { target tags }\end{array}$ \\
\hline 2 & 6 & 36 \\
3 & 6 & 36 \\
4 & 6 & 23 \\
5 & 6 & 22 \\
6 & 6 & 18 \\
\hline \hline
\end{tabular}

The main steps of the proposed methodology for the feature extraction are summarized by means of the flow chart in Fig. 13.

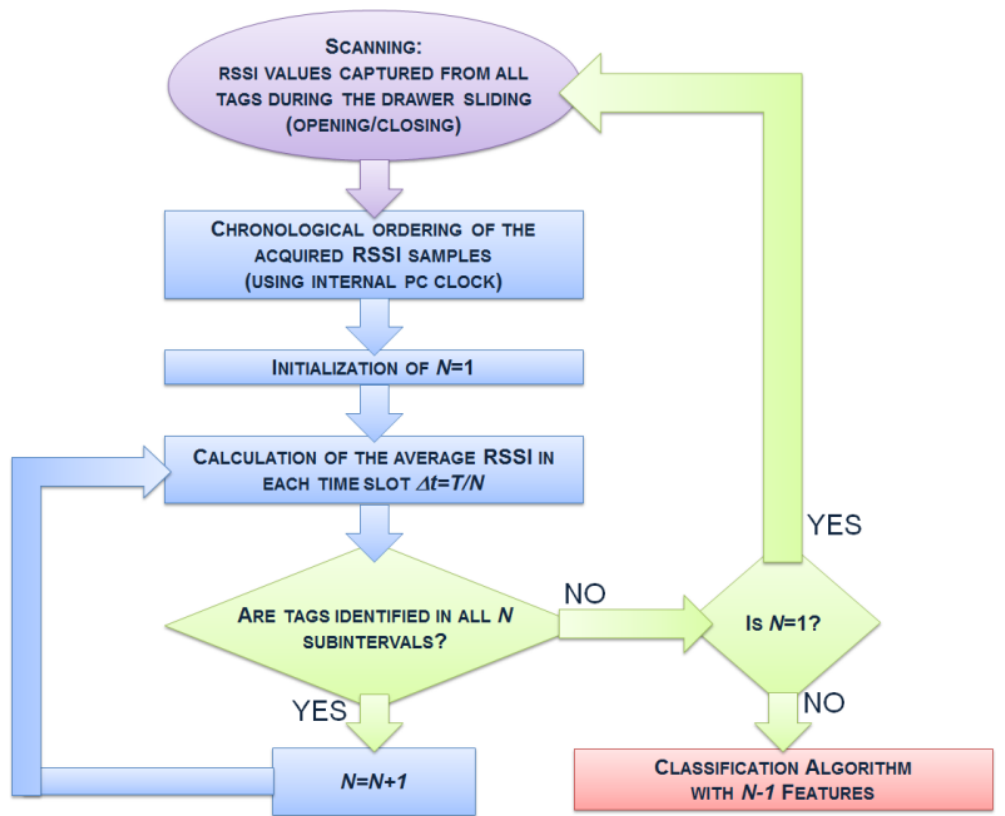

Fig. 13 Flow chart of the proposed methodology exploiting drawer sliding actions.

The overall accuracy is represented in Fig. 14 through a bar chart, for seven repeated tests that were obtained for different target and reference tag distributions within the drawer. $N=3$ features were considered by employing both the LDA and the Naïve-Bayes algorithms. For a drawer sliding interval of $T=13 \mathrm{~s}$ (four test cases), an average overall accuracy $\overline{\mathrm{OA}}=0.98$ and $\overline{\mathrm{OA}}=0.96$ is reached for the LDA algorithm and the Naïve-Bayes algorithm, respectively. By using a drawer sliding interval $T=5 \mathrm{~s}$ (three test cases), the average overall accuracy decreases to $\overline{\mathrm{OA}}=0.90$ and $\overline{\mathrm{OA}}=0.92$ for the LDA and Naïve-Bayes algorithm, respectively. Above results confirm that the set of features extracted from RSSI samples collected during drawer sliding is so effective that a satisfactory classification can be obtained independently from the employed classification algorithm. LDA algorithm performance is dependent on the number of reference tags and it was demonstrated that at least two reference tags for each drawer region are needed. 


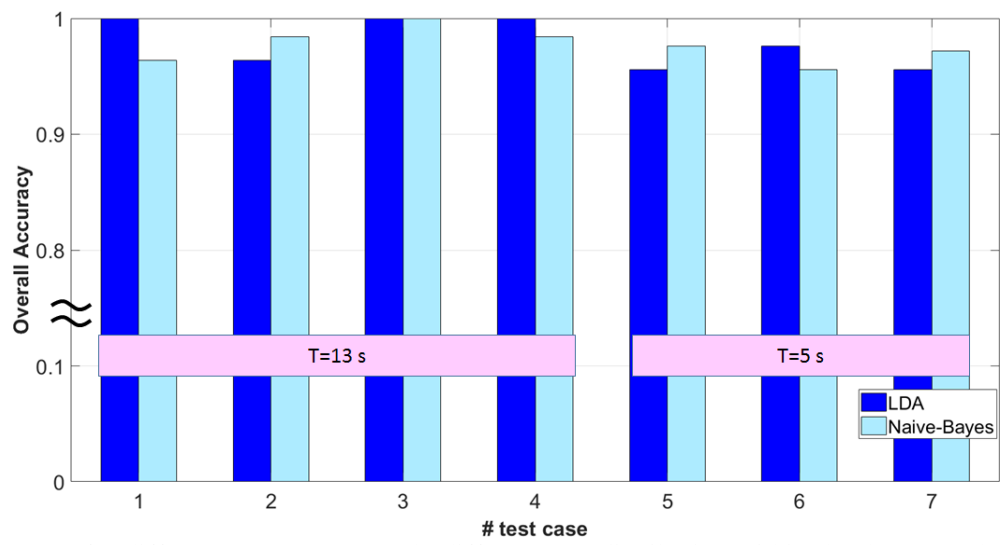

Fig. 14 Bar chart of the overall accuracy for different test cases (namely different tags distribution within the drawer and various sliding intervals) as result of LDA algorithm (dark bar) and Naïve-Bayes algorithm (light bar) by using $N=3$ features, $M=2$ drawer regions, thirty-six target tags, six reference tags (three for each region).

Although just a basic implementation was considered for both classification algorithms, the achieved performance $(\overline{O A}>0.90)$ confirmed the robustness of the proposed approach. It is expected that similar performance can be achieved with other classification algorithms. Mainly, the basic idea is to collect several RSSI data during the drawer sliding actions, namely by varying the relative distance between reader antenna and tags with no additional cost.

The method is shown just for one sliding drawer but it can be applied to each drawer within the chest; in such case, one reader antenna for each drawer is required.

Since the classification decision is actually based on a comparison between the RSSI profile of the detected tag and the RSSI profile of the reference tags, that are all acquired during the same sliding operation, in principle the method may work independently on the sliding speed and spatial sliding interval. On the other hand, a robust and effective classification method requires for as large as possible spatial sliding intervals and enough RSSI samples. Above requirements imply that the maximum drawer aperture should be exploited, with a relatively low sliding speed. Moreover, to limit the complexity of the preprocessing algorithm devoted to filter the raw RSSI values for getting the classification features, a constant and relatively low speed is preferred (while knowing the exact speed value is not required at all).

Finally, it is worth mentioning that the effect of package materials was taken into account in the experimental analysis since tags were directly applied to drug boxes, while the effect of liquids or solids inside the boxes was not considered. Although item material can adversely affect tag performance in terms of sensitivity, it is expected that tag readability is not so critical since the reader antenna is very close to the tagged boxes. The proposed method assumes tags readability; how to guarantee such readability is out the scope of this work.

\section{CONCLUSION}

A selective measurement method for UHF-RFID tag classification in smart drawer applications was presented. The RSSI values are used as features of a stochastic classification algorithm. The cases of multiple static reader antennas and a single reader antenna measuring during usual opening/closing operations were both investigated. RSSI measurements were acquired by a commercial reader, and the well-known supervised LDA and Naïve-Bayes classification algorithms were employed. The achieved tag classification accuracies confirm the validity of the measurement method to determine the tag belonging region in an RFID-based smart drawer. Finally, it was found that the method based on a single antenna exploiting the drawer natural sliding movements can guarantee classification performance comparable to that achievable with multiple reader antennas, while representing a cost-effective and easy-to-implement practical solution. It was underlined that the drawer opening and closing actions are natural operations when storing or getting tagged objects, so the proposed classification technique does not require any extra effort.

\section{REFERENCES}

[1] R. H. Murofushi, J. J. P. Z. S. Tavares, "Towards fourth industrial revolution impact: smart product based on RFID technology", IEEE Instrum. Meas. Mag., vol. 20, no. 2, pp. 51-56, Apr. 2017.

[2] L. Catarinucci et al., "An IoT-Aware Architecture for Smart Healthcare Systems", IEEE Internet Things J., vol. 2, no. 6, pp. 515-526, Dec. 2015.

[3] S. Amendola, R. Lodato, S. Manzari, C. Occhiuzzi, and G. Marrocco, "RFID Technology for IoT-Based Personal Healthcare in Smart Spaces", IEEE Internet Things J., vol. 1, no. 2, pp. 144-152, Apr. 2014.

[4] G. M. Gaukler, "Item-Level RFID in Retail Supply Chain With Stock-Out-Based Substitution", IEEE Trans. Ind. Informat., vol. 7, no. 2, pp. 326-370, May 2011.

[5] A. Soylemezoglu, M. J. Zawodniok, and S. Jagannathan, "RFID-Based Smart Freezer", IEEE Trans. Ind. Electron., vol. 56, no. 7, pp. 2347-2356, Jul. 2009.

[6] A. Buffi, P. Nepa, and F. Lombardini, “A Phase-Based Technique for Localization of UHF-RFID Tags Moving on a Conveyor Belt: Performance Analysis and Test-Case Measurements”, IEEE Sensors J., vol. 15, no. 1, pp. 387-396, Jan. 2015. 
[7] M. Scherhäufl, M. Pichler, and A. Stelzer, "UHF RFID Localization Based on Phase Evaluation of Passive Tag Arrays", IEEE Trans. Instrum. Meas., vol. 64, no. 4, pp. 913-922, Apr. 2015.

[8] P. Nazemzadeh, F. Moro, D. Fontanelli, D. Macii, and L. Palopoli, "Indoor Positioning of a Robotic Walking Assistant for Large Public Environments", IEEE Trans. Instrum. Meas., vol. 64, no. 11, pp. 2965-2976, Nov. 2015.

[9] A. A. N. Shirehjini, A. Yassine, and S. Shirmohammadi, "An RFID-Based Position and Orientation Measurement System for Mobile Objects in Intelligent Environments”, IEEE Trans. Instrum. Meas., vol. 61, no. 6, pp. 1664-1675, Jun. 2012.

[10] R. Miesen, F. Kirsch, and M. Vossiek, "UHF RFID Localization Based on Synthetic Apertures", IEEE Trans. Autom. Sci. Eng., vol. 10, no. 3, pp. 807-815, Jul. 2013.

[11] Y. Rekik, E. Sahin, Y. Dallery, “Analysis of the Impact of the RFID Technology on Reducing Product Misplacement Errors at Retail Stores", Int. J. Prod. Econ., vol. 112, no. 1, pp. 264-278, Mar. 2008.

[12] H.-J. Wang, G. Wang, and Y. Shu, "Design of RFID Reader Using Multi-Antenna with Difference Spatial Location", in Proc. Int. Conf. Wireless Commun., Netw. Mobile Comput., Sep. 2007, pp. 2070-2073.

[13] A. Michel, R. Caso, A. Buffi, P. Nepa, and G. Isola, "Meandered TWAs array for Near-Field UHF RFID applications", Electron. Lett., vol. 50, no. 1, pp. 17-18, Jan. 2014.

[14] L. Catarinucci, et al., "Smart RFID Antenna System for Indoor Tracking and Behavior Analysis of Small Animals in Colony Cages", IEEE Sensors J., vol. 14, no. 4, pp. 1198-1206, Apr. 2014.

[15] A. Michel and P. Nepa, "UHF-RFID Desktop Reader Antennas: Performance Analysis in the Near-Field Region", IEEE Antennas Wireless Propag. Lett., vol. 15, pp. 1430-1433, 2016.

[16] A. Buffi, A. Michel, R. Caso, and P. Nepa, "Near-field coupling in UHF-RFID systems", in Proc. Int. Symp. Electromagn. Theory, Hiroshima, May 2013, pp. 408-411.

[17] J. S. Choi, L. Hyun, D. W. Engels, and R. Elmsari, "Passive UHF RFID-Based Localization Using Detection of Tag Interference on Smart Shelf", IEEE Trans. Syst., Man, Cybern. C, Appl. Rev., vol. 42, no. 2, pp. 268-275, Mar. 2012.

[18] Y. Yuan and D. Yu, "UHF RFID shelf solution with cascaded reader antenna and positioning capability", in Proc. Int. Conf. RFID, Apr. 2012, pp. 149-156.

[19] P. Pivato, L. Palopoli, and D. Petri, "Accuracy of RSS-Based Centroid Localization Algorithms in an Indoor Environment", IEEE Trans. Instrum. Meas., vol. 60, no. 10, pp. 3451-3460, Oct. 2011.

[20] V. Pasku, M. L. Fravolini, and A. Moschitta, "Effects of antenna directivity on RF ranging when using space diversity techniques", Measurement, vol. 98, pp. 429-438, Feb. 2017.

[21] A. D'Alessandro, A. Buffi, P. Nepa, and G. Isola, "A localization technique for smart bookshelves based on UHF-RFID systems", in Proc. IEEE Antennas Propag. Soc. Int. Symp. (APSURSI), Orlando, FL, USA, Jul. 2013, pp. 1112-1113.

[22] A. Buffi and P. Nepa, "The SARFID Technique for Discriminating Tagged Items Moving Through a UHF-RFID Gate", IEEE Sensors J., vol. 17, no. 9, pp. 2863-2870, May 2017.

[23] R. San-Segundo, J. D. Echeverry-Correa, C. Salamea, and J. M. Pardo, "Human activity monitoring based on hidden Markov models using a smartphone", IEEE Instrum. Meas. Mag., vol. 19, no. 6, pp. 27-31, Dec. 2016.

[24] G. Betta, D. Capriglione, M. Gasparetto, E. Zappa, C. Liguori, and A. Paolillo, "Face recognition based on 3D features: Management of the measurement uncertainty for improving the classification", Measurement, vol. 70, pp. 169-178, Jun. 2015.

[25] M. Pardo and G. Sberveglieri, "Coffee Analysis With an Electronic Nose", IEEE Trans. Instrum. Meas., vol. 51, no. 6, pp. 1334-1339, Dec. 2002.

[26] A. D'Alessandro, A. Buffi, and P. Nepa, "RFID-Based Smart Shelving Storage Systems", in Proc. Asia-Pacific Microw. Conf. (APMC), Dec. 2012, pp. 1034-1036.

[27] K. Finkenzeller, RFID Handbook: Fundamentals and Applications in Contactless Smart Cards and Identification, 2nd ed. New York, NY, USA: Wiley, 2003.

[28] P. V. Nikitin, K. V. S. Rao and R. D. Martinez, "Differential RCS of RFID tag”, Electron. Lett., vol. 43, no. 8, pp. 431-432, Apr. 2007.

[29] A. Michel, A. Buffi, P. Nepa and G. Manara, "Antennas for UHF-RFID printer-encoders", in Proc. IEEE 15th Medit. Microw. Symp. (MMS), Lecce, Italy, Nov./Dec. 2015, pp. 1-4.

[30] C. A. Balanis, Antenna Theory: Analysis and Design, vol. 1. Hoboken, NJ, USA: Wiley 2005.

[31] P. V. Nikitin and K. V. S. Rao, "Antennas and Propagation in UHF RFID Systems", in Proc. IEEE Int. Conf. RFID, Las Vegas, NV, Apr. 2008, pp. 277288.

[32] M. Scherhäufl, M. Pichler and A. Stelzer, "UHF RFID Localization Based on Evaluation of Backscattered Tag Signals", IEEE Trans. Instrum. Meas., vol. 64, no. 11, pp. 2889-2899, Nov. 2015.

[33] Y. Zhao, K. Liu, Y. Ma, Z. Gao, Y. Zang, and J. Teng, "Similarity Analysis-Based Indoor Localization Algorithm With Backscatter Information of Passive UHF RFID Tags", IEEE Sensors J., vol. 17, no. 1, pp. 185-193, Jan. 2017.

[34] J.S. Bilodeau, A. Bouzouane, B. Bouchard, and S. Gaboury, "An experimental comparative study of RSSI-based positioning algorithms for passive RFID localization in smart environments", J. Ambient Intell. Hum. Comput., vol. 12652, pp. 1-17, Jun. 2017.

[35] A. S. Paul and E. A. Wan, "RSSI-Based Indoor Localization and Tracking Using Sigma-Point Kalman Smoothers", IEEE J. Sel. Topics Signal Process., vol. 3, no. 5, pp. 860-873, Oct. 2009.

[36] C. Qiu and M. W. Mutka, "Cooperation among smartphones to improve indoor position information", in Proc. IEEE 16th Int. Symp. World Wireless, Mobile Multimedia Networks (WoWMoM), Boston, MA, USA, Jun. 2015, pp. 1-9.

[37] W. J. Krzanowski, Principles of Multivariate Analysis: A User's Perspective. New York, NY, USA: Oxford Univ. Press, 1988.

[38] X. Wu et al. "Top 10 algorithms in data mining", Knowl. Inf. Syst., vol. 14, no. 1, pp. 1-37, 2008

[39] R. G. Congalton and K. Green, Assessing the Accuracy of Remotely Sensed Data: Principles and Practices. Boca Raton, FL, USA: CRC Press, 1999.

[40] T. Fujita, Y. Numata, T. Yoshimi, M. Mizukawa, and Y. Ando, "A study of intelligent drawer with RFID tag information reading system for Intelligent Space", in Proc. Int. Conf. Mechatronics Autom., Aug. 2012, pp. 533-538.

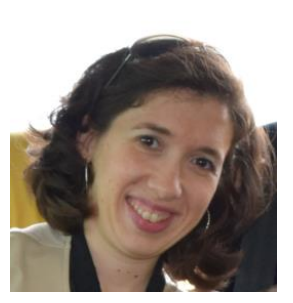

Alice Buffi (S'10-M'12) received the bachelor's and master's (summa cum laude) degrees in telecommunications engineering with Percorso di Eccellenza and the Ph.D. degree in applied electro-magnetism in electrical and biomedical engineering, electronics, smart sensors, nanotechnologies with Doctor Europaeus label from the University of Pisa, Pisa, Italy, in 2006, 2008, and 2012, respectively. She joined the Queen Mary University of London, London, U.K. a Visiting Ph.D. Student in 2012. Since 2012, she has been with the University of Pisa, where she is currently an Assistant Professor with the Department of Energy, Systems, Territory and Construction Engineering. She co-authored several international journal papers and international conferences contributions and holds a European patent. Her research interests include the analysis and design of near-field focused planar microstrip arrays, microstrip antennas design, near-field coupling in UHF-radio frequency identification (RFID) systems, wireless power transfer and measurement methods for localization and classification in RFID-based smart spaces.

Dr. Buffi received of the Young Scientist Award from the International Union of Radio Science, Commission B, in 2013 and 2016. 


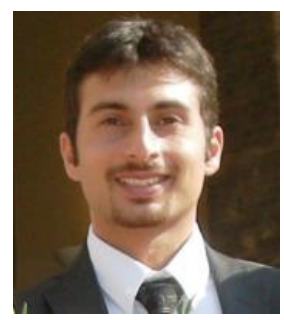

Andrea Michel (S'08-M'15) received the B.E., M.E. (summa cum laude) and PhD degrees in telecommunications engineering from the University of Pisa, Pisa, Italy, in 2009, 2011 and 2015, respectively.

Since 2015 he has been a Post-Doctoral Researcher in applied electromagnetism at the Microwave and Radiation Laboratory, Department of Information Engineering, University of Pisa, focusing on the design of integrated antenna for communication systems and smart antennas for near-field UHF-radio frequency identification (RFID) readers. In 2014, he joined the ElectroScience Laboratory, The Ohio State University, Columbus, OH, USA, as a Visiting Scholar, where he has been involved in research on a theoretical analysis on the accuracy of a novel technique for deep tissue imaging. His current research interests include the design of antennas for automotive applications, multiple-in multiple-out systems, and wearable communication systems, in collaboration with other research institutes and companies.

Dr. Michel received the Young Scientist Award from the International Union of Radio Science, Commission B, in 2014, 2015 and 2016 and the Best Paper Honorary Mention from the IEEE International Conference on RFID Technology and Applications, Shunde, Guangdong, China, in 2016.

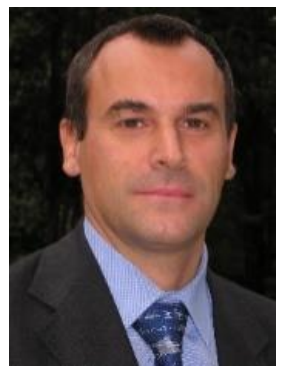

Paolo Nepa (M’07) received the Laurea (Doctor) degree (summa cum laude) in electronics engineering from the University of Pisa, Italy, in 1990.

Since 1990, he has been with the Department of Information Engineering, University of Pisa, where he is currently an Associate Professor. In 1998, he was at the Electro Science Laboratory (ESL), The Ohio State University (OSU), Columbus, OH, as a Visiting Scholar supported by a grant of the Italian National Research Council. At the ESL, he was involved in research on efficient hybrid techniques for the analysis of large antenna arrays. His research interests include the extension of high-frequency techniques to electromagnetic scattering from material structures and its application to the development of radio propagation models for indoor and outdoor scenarios of wireless communication systems, the design of wideband and multiband antennas, mainly for base stations and mobile terminals of communication systems, the design of antennas optimized for near-field coupling and focusing, channel characterization, and wearable antenna design and diversity scheme implementation, for body-centric communication systems. In the context of radio frequency identification (RFID) systems, he focuses on techniques and algorithms for UHF-tag localization and RFID-based smart shelves.

Dr. Nepa received the Young Scientist Award from the International Union of Radio Science, Commission B, in 1998. He is an Associate Editor of the IEEE ANTENNAS AND WIRELESS PROPAGATION LETTERS.

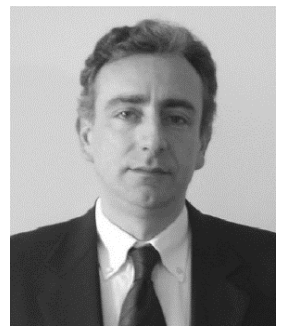

Bernardo Tellini (M’05) was born in Pisa, Italy, in 1969. He received the M.S. (Laurea) and Ph.D. degrees in electrical engineering from the University of Pisa, Pisa, in 1993 and 1999, respectively.

Since 2000, he has been with the University of Pisa, where he is currently a Full Professor of electrical measurements with the Department of Energy, Systems, Territory and Construction Engineering. His current research interests include characterization of magnetic materials metamaterials and ageing process in battery cells, electromagnetic emission associated with fast electromagnetic transients, sensors and transducers for pulsed power applications, and magnetohydrodynamics problems.

Dr. Tellini is a member of the IEEE Instrumentation and Measurement Society and the IEEE Magnetics Society. He is the Vice Chair of the IEEE Instrumentation and Measurement Society Italian Chapter and the IEEE Italy Section. He was the Chair of European Pulsed Power Laboratories from 2010 to 2014, and the General Chair of the International Instrumentation and Measurement Technology Conference in 2015. 